T5 TOWARDS HUMAN LUNG REGENERATION IN ENDSTAGE RESPIRATORY FAILURE: GENETICALLYMODIFIABLE 3D ORGANOID CULTURE OF HUMAN EMBRYONIC LUNG STEM CELLS ENABLES FOR THE FIRST TIME THE STUDY OF HUMAN LUNG DEVELOPMENT IN VITRO

${ }^{1} \mathrm{MZ}$ Nikolic, ${ }^{1} \mathrm{~J} A$ Johnson, ${ }^{1} \mathrm{D}$ Sun, ${ }^{1} \mathrm{O}$ Caritg, ${ }^{1} \mathrm{U}$ Laresgoiti, ${ }^{1} \mathrm{~J}$ Brady, ${ }^{1} \mathrm{G}$ Allen, ${ }^{2} \mathrm{~A}$ Giangreco, ${ }^{1} \mathrm{EL}$ Rawlins. 'Gurdon Institute, University of Cambridge, Cambridge, Cambridgeshire; ${ }^{2}$ University College London, London, UK

\subsection{6/thoraxjnl-2016-209333.5}

Introduction and objectives Regeneration of healthy lung tissue in patients with end-stage respiratory disease (ESRD) would cure disease, rather than treating symptoms. For this a detailed understanding of lung development is needed and the mouse has been used extensively as an in vivo genetically-modifiable model. Differentiation and validation of human induced pluripotent stem cells (hiPSCs) is entirely based on mouse literature. The most important epithelial stem cell population in developing lungs is found in distal branching tips, and these Sox9+ lung epithelial stem cells (LESCs) generate all epithelial lineages. Our objective was to develop a self-renewing, genetically-modifiable epithelial in vitro culture system from human embryonic LESCs and differentiate them into alveolar and bronchiolar cells.

Methods Human embryonic LESCs were characterised using genome-wide transcriptional analysis (RNAseq) and immunohistochemistry (5-20 post-conceptional weeks). LESCs were microdissected and self-renewing expansion in 3D organoid culture was established empirically. Using RNAseq and immunohistochemistry, we assessed the similarities between cultured and fresh LESCs. Genetic stability was evaluated by karyotyping. Organoids were differentiated in vitro, or in vivo using xenotransplantation into bleomycin-injured adult mouse lungs or kidney capsule. Gene editing was done using CRISPR-Cas9 to delete SOX9.

Results RNAseq of LESCs identified broad-scale transcriptional differences between mouse and human embryonic lung stem cells. Human LESCs were successfully expanded for over 10 months as karyotypically-stable 3D organoids using a combination of 7 signalling molecules. The LESC stem cell markers, transcriptome and organoid morphology were maintained throughout the culture period. Bronchiolar and alveolar differentiation was achieved in vitro and in vivo. Moreover, xenotransplantation of organoids into bleomycin-injured adult mouse lungs was extremely efficient. Knocking out SOX9 led to a loss-of-selfrenewing phenotype.

Conclusions Our novel genetically-modifiable human embryonic lung culture system enables for the first time the in vitro study of human lung development and disease modelling. We anticipate that this work will transform lung regenerative medicine by guiding the development of improved protocols for hiPSC differentiation and manipulation of adult stem cells in vivo, with benefits for patients with ESRD. Additionally, our protocol for xenotransplantation following lung injury provides the first method for efficient future cell therapy.

\section{T6 RANDOMISED SHAM-CONTROLLED TRIAL OF TRANSCUTANEOUS ELECTRICAL STIMULATION IN OBSTRUCTIVE SLEEP APNOEA}

${ }^{1} \mathrm{M}$ Pengo, ${ }^{2} \mathrm{X}$ Sichang, ${ }^{3} \mathrm{C}$ Ratneswaran, ${ }^{4} \mathrm{~N}$ Shah, ${ }^{3} \mathrm{~K}$ Reed, ${ }^{5} \mathrm{~T}$ Chen, ${ }^{5} \mathrm{~A}$ Douiri, ${ }^{4} \mathrm{~N}$ Hart, ${ }^{2}$ Y Luo, ${ }^{3} \mathrm{G}$ Rafferty, ${ }^{1} \mathrm{GP}$ Rossi, ${ }^{4} \mathrm{~A}$ Williams, ${ }^{6} \mathrm{Ml}$ Polkey, ${ }^{3} \mathrm{~J}$ Moxham, ${ }^{4} \mathrm{~J}$ Steier. ${ }^{1}$ University of Padua, Department of Medicine (DIMED), Padua, Italy; ${ }^{2}$ State Key Laboratory of Respiratory Disease, The First Affiliated Hospital of Guangzhou Medical University, Guangzhou, China; ${ }^{3}$ King's College London, Faculty of Life Sciences and Medicine, London, UK; ${ }^{4}$ Guy's and St Thomas' NHS Foundation Trust, Lane Fox Respiratory UnitSleep Disorders Centre, London, UK; ${ }^{5}$ King's College London, Division of Health and Social Care, London, UK; ${ }^{6}$ NIHR Respiratory Biomedical Research Unit at the Royal Brompton and Harefield NHS Foundation Trust and Imperial College, London, UK

\subsection{6/thoraxjnl-2016-209333.6}

Obstructive sleep apnoea is characterised by a loss of neuromuscular tone of the upper airway dilator muscles while asleep. Continuous positive airway pressure is a well-established long-term treatment, but patient compliance is limited. This study investigated the effectiveness of transcutaneous electrical stimulation in patients with obstructive sleep apnoea.

This was a randomised, sham-controlled cross-over trial using transcutaneous electrical stimulation of the upper airway dilator muscles in patients with confirmed obstructive sleep apnoea. Patients were randomly assigned to two nights, sham stimulation and active treatment. The primary outcome was the $4 \%$ oxygen desaturation index, responders were defined as patients with a reduction $>25 \%$ in the oxygen desaturation index when compared to sham stimulation and/or with an index $<5 /$ hour in the active treatment night.

In 36 patients (age mean 50.8 (standard deviation 11.2) years, male/female 30/6, body-mass-index median 29.6 (interquartile range 26.9-34.9) kg/m², Epworth Sleepiness Scale 10.5 (4.6) points, oxygen desaturation index median 25.7 (16.0-49.1)/hour, apnoea hypopnoea index median 28.1 (19.0-57.0)/hour) the primary outcome measure improved when comparing sham stimulation (median $26.9(17.5-39.5)$ /hour) to active treatment (median $19.5(11.6-40.0)$ /hour; $p=0.026)$, a modest reduction of the mean by 4.1 (95\% CI: $-0.6-8.9) /$ hour. Secondary outcome parameters of patients' perception indicated that stimulation was well tolerated. Responders (47.2\%) were predominantly from the mild-moderate obstructive sleep apnoea category. In this subgroup, the oxygen desaturation index was reduced by $10.0(95 \%$ CI: 3.9-16.0)/hour $(\mathrm{p}<0.001)$ and the apnoea hypopnoea index was reduced by 9.1 (95\% CI: 2.0-16.2)/hour ( $p=0.004)$.

Transcutaneous electrical stimulation of the pharyngeal dilators during a single night in patients with obstructive sleep apnoea improves upper airway obstruction and is well tolerated. (TESLA trial registration at NCT01661712) 


\section{The Difficult Asthma Patient}

\section{S1 BIOMARKERS IN ADULT ASTHMA: A SYSTEMATIC REVIEW OF 8-ISOPROSTANE IN EXHALED BREATH CONDENSATE}

${ }^{1} \mathrm{AM}$ Peel, ${ }^{1} \mathrm{CJ}$ Crossman-Barnes, ${ }^{1} \mathrm{~J}$ Tang, ${ }^{2} \mathrm{SJ}$ Fowler, ${ }^{3} \mathrm{GA}$ Davies, ${ }^{1} \mathrm{AM}$ Wilson, ${ }^{1}$ YK Loke. ${ }^{1}$ University of East Anglia, Norwich, UK; ${ }^{2}$ University Hospital South Manchester, Manchester, UK; ${ }^{3}$ Swansea University, Swansea, UK

\subsection{6/thoraxinl-2016-209333.7}

Introduction The potential of exhaled breath condensate (EBC) as a non-invasive indicator of airways disease has been studied for three decades or more. 8-isoprostane is a product of lipid peroxidation which can be detected within EBC. Studies have reported this as a potential objective indicator of oxidative stress in asthma. We therefore aimed to assess the evidence for the use of 8 -isoprostane in exhaled breath condensate (EBC) as a biomarker in adult asthma.

Design A systematic review and meta-analysis of EBC 8-isoprostane in asthma.

Methods We searched a number of online databases (including PubMed, Embase and Scopus) in January 2016. We included studies of adult non-smokers with EBC collection and asthma diagnosis conducted according to recognised guidelines. We aimed to pool data using random effects meta-analysis and assess heterogeneity using I2. Study quality and risk of bias was assessed using QUADAS-2 and GRADE.

Results We included twenty studies, the findings from which were inconsistent. Seven studies $(\mathrm{n}=329)$ reported 8-isoprostane concentrations in asthma to be significantly higher than that of control groups, whilst six studies $(n=403)$ did not. Only four studies had results appropriate for inclusion in a random effects meta-analysis of mean difference between asthma and controls (see Figure 1). This found a statistically significant betweengroups difference of $+22 \mathrm{pg} / \mathrm{ml}$ in asthma.

Confidence in the result is limited by the small number of studies; by substantial methodological and statistical heterogeneity $\left(\mathrm{I}^{2}=94\right)$; and by an inability to assess the risk of bias in key domains of the quality assessment tool.

Conclusion The clinical value of EBC 8-isoprostane as a quantitative assessment of oxidative stress in asthma remains unclear due to variability in results and methodological heterogeneity. It will be essential to develop accurate, reliable and standardised methods of both EBC collection and 8-isoprostane analysis if its use as a biomarker in asthma is to be evaluated.
S2 FRACTIONAL EXHALED NITRIC OXIDE (FENO) SUPPRESSION TO IDENTIFY NON-ADHERENCE IN DIFFICULT ASTHMA

${ }^{1} \mathrm{KJ}$ Hetherington, ${ }^{2} \mathrm{RW}$ Costello, ${ }^{1} \mathrm{LG}$ Heaney. 'Queens University Belfast, Belfast, UK; ${ }^{2}$ Royal College of Surgeons Ireland, Dublin, Ireland

\subsection{6/thoraxjnl-2016-209333.8}

Introduction Non-adherence, both intentional and non-intentional, is prevalent in 'difficult to manage' asthma and identification of patients who should respond to inhaled corticosteroids (ICS) is essential to reduce exacerbation risk and improve asthma control, but also to prevent inappropriate escalation of treatment (oral corticosteroids or biological therapies). Composite biomarker assessments (FeNO and blood eosinophils) have been shown to predict exacerbation risk in patients with asthma and these biomarkers also predict response to steroid treatment. It has previously been demonstrated that suppression of FeNO with directly observed ICS over 7 days can identify non-adherence to ICS treatment. The aim of this work was to further validate this test across UK severe asthma centres in the RASP-UK Consortium and to examine if the test can identify patients who should achieve good asthma control with better adherence to ICS treatment.

Methods Using remote monitoring technology (Vitalograph INCA $^{\mathrm{TM}}$ device and Aerocrine NIOX Vero), we developed a webbased interface to deliver FeNO suppression testing in RASP-UK Severe Asthma Centres. We examined the utility of FeNO suppression testing to predict inhaled steroid responsiveness and composite biomarker profile after 30 days of monitored optimised treatment on standard high dose ICS/LABA.

Results Forty of seventy patients had a positive suppression test. Using information from the Vitalograph INCA $^{\text {TM }}$ server to highlight technique and timing errors, these issues were addressed and patients proceeded to 30-day monitoring of high dose ICS/ LABA. A positive FeNO suppression test (Figure A) identified a biomarker-low population when adherent with high dose ICS ( $\geq 80 \%$ of inhaled steroid) whereas a negative test (Figure B) identified a biomarker-high population when adherent with high dose ICS.

Conclusion FeNO suppression testing is an effective means of identifying non-adherence to inhaled steroids in patients with difficult to manage asthma. This test can identify patients who should be optimised on inhaled steroids prior to consideration of treatment escalation.

Collaborating Centres Queen's University Belfast, University of Leicester, University of Glasgow, University of Oxford, The

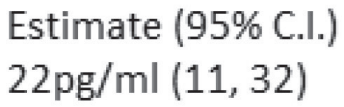

$1^{2}=94$

$P<0.001$

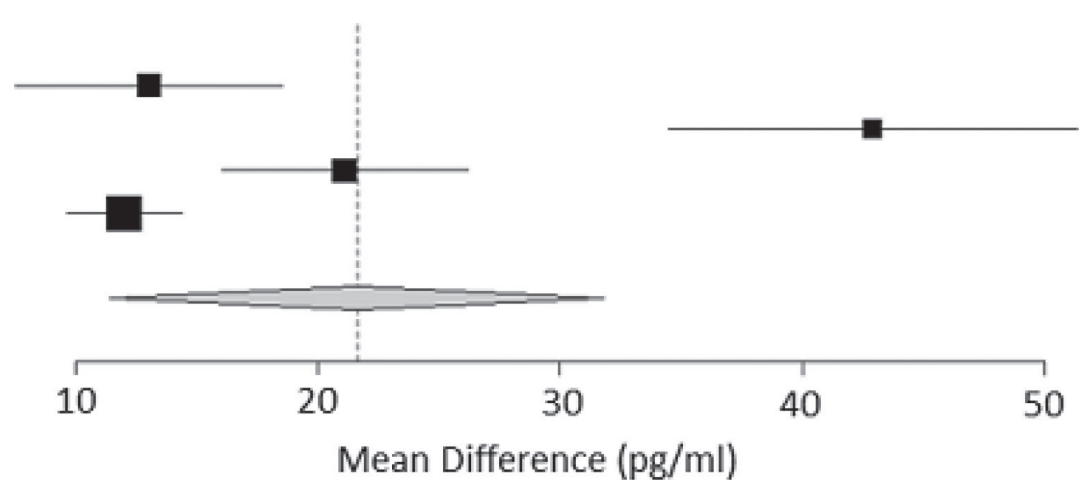

Mean Difference $(\mathrm{pg} / \mathrm{ml})$

Abstract S1 Figure 1 Random effects meta-analysis of mean between-group difference (asthma vs controls) 